\title{
Opportunistic Communication in Highly Congested and Dynamic Urban Areas
}

\author{
Hayder Al-Ghanimi, Milena Radenkovic, Alaa Hassan \\ University of Babylon, University of Nottingham, University of Kirkuk
}

\begin{abstract}
This paper explores feasibility and performance characteristics of delay tolerant data dissemination in highly congested and dynamic urban area. We look into London scenario during the Olympic Games as highly challenging as it has significantly increased traffic and communication demands added over a short period of time to the already saturated and densely populated city. We investigate two different mobility scenarios that we run on the map of London Olympic area, a Random Movement Scenario and Work Day Movement Scenario, to test how effective opportunistic data transmission can be. We focus on intelligent probabilistic forwarding mechanisms and compare them to other state of the art delay tolerant protocols across a range of metric such as the number of delivery success, latency and overheads. Our results show that Spray and Wait has the best performance with the Random Scenario but the worst performance with the Work Day Movement Scenario whereas the intelligent probabilistic protocols has the lowest with Random Scenario but it gives better performance than Spray and Wait in Work Day Movement Scenario.
\end{abstract}

\section{INTRODUCTION}

Current mobile (handheld) devices provide valuable opportunities for creating cheap networks by connecting the devices in ad hoc manner and utilising a range of wireless technologies. Even though delay tolerant and asynchronous, these networks can be used in a range of application scenarios ranging from bulk data transfers to multimedia streaming data. These kinds of networks are particularly important in highly congested areas, such as during the events where mass number of people gather for a short time in one location (e.g. World Cup or Olympics) and expect to communicate over their devices continuously. This can cause telephone networks or any infrastructure nodes to get congested and become unusable. When this happens, wireless users devices can self organise to build an ad hoc network that moves the traffic away from the infrastructure an into the infrastrcurless mode by transmitting messages to their neighbour and acting as mobile routers. These protocols have different probabilities for delivering the messages to the destination nodes and need to consider a range of resource parameters such as energy and storage space of nodes that can quickly get used up if a node is very central/popular and many other nodes use it to receive and send their data.

We have explored a range of stare of the art protocols in this new and very challangeing scenario such as [2], [3], and [4]. [2] Works by sending the message to a specific number of nodes and then waiting until the nodes have direct access to the destination node in order to hand the message to it. In [3], every node sends messages to any other nodes it meets. Only in [3], the message forwarding in base don on the intelligent analysis of historical encounters between the nodes and predication of their future encounters and locations. [3] is very useful in scenarios of social mobile networks because people do not normally move randomly, but they often go to specific locations at regular times, such as houses and offices. In this case every node performs statistical analysis for each encounter it meets, calculates probabilities of each node's delivery probability and forwards the message to the node with the highest delivery probability. It is this last protocol whose performance we are aiming to analyse in more depth. We use ONE [5.6] with a realistic map of London Olymics area during th eOlymic games and explore two mobility scenarios: Work Day Movement Scenario and Random Movement Scenario. We have designed two application scenarios to define the number of nodes in the map and their sending and receiving behaviour. We compare three protocols for a range of scenarios and across a range of metrics.

\section{RELATED WORK}

The area of delay tolerant and opportunistic data communication is a very active research area and there are many protocols proposed. This section briefly reviews few of the major ones.

\section{A. Prophet Routing}

Lindgren, Doria and Schelén identify probabilistic routing in intermittently connected networks called the Prophet protocol [4]. This protocol is the protocol which we are going to focus on. The Prophet routing protocol uses the history of encounters between nodes to inform its decision to forward a message to the next hop. It does this when any two nodes use the same protocol (Prophet), they exchange information and make 
some calculations like updating the delivery probability value for connecting nodes. The first thing that happens is to check whether this node is a destination node for the message. Otherwise, three calculations for updating the delivery probability value are started. At the beginning, updating the metric for connecting nodes is required at this level. Then, aging the nodes that do not encounter the current node within a certain time means that this node is not beneficial in helping the message reach the destination node. Furthermore, updating the transitive property for delivery predictability should be done. Therefore, the node that has high delivery predictability is a good node to which to transfer the message, so the transmission of the message is based on delivery predictability. In other words, the nodes, which have high delivery predictability, would be chosen.

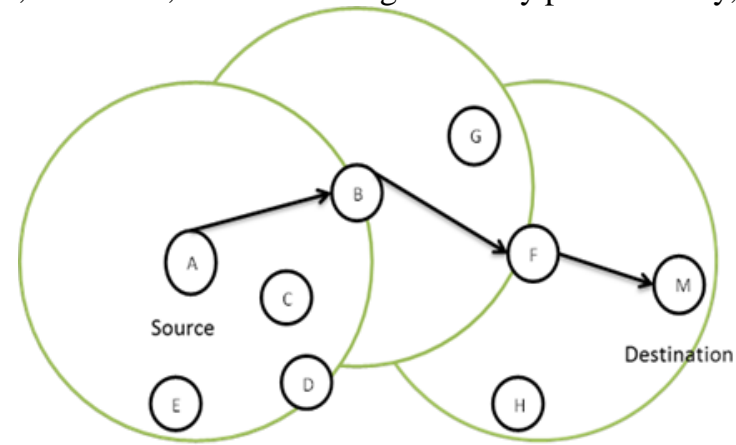

Fig. 1 Sample for Message Path by using Prophet Routing Protocol

As can be seen from the Figure [1], the source node, A, wants to send a message to node $\mathrm{M}$, so it generates the message, then node A forwards the message to the highest delivery node B from its table of delivery predictabilities for the available nodes to forward to. After that, node B checks whether the destination node is available to forward the message. If it is not available, the message would be forwarded to the highest delivery predictability node F from the table of B. Finally, node F checks around nodes to see whether the destination node is available which is $\mathrm{M}$, so it forwards the message to node $\mathrm{M}$.

Lindgren, Doria and Schelén, however, simulate their protocol on random scenarios and small community scenarios which they have designed. However, they concluded that the ONE simulation has shown that in the community based scenario with Prophet routing gives better performance compared with Epidemic Routing. Furthermore, the results from the ONE simulator for the random scenario using Prophet routing still gives better performance than Epidemic Routing, although Prophet is not designed for random environments [6].

\section{B. Spray and Wait Routing}

Spyropoulos, Psounis and Raghavendra analyse the Spray and Wait Routing protocol which is one of the Delay-Tolerant Networking DTN routing protocols in intermittently connected networks [2]. It combines the benefit of both epidemic, speed with the simplicity, routing and thriftiness of direct transmission. In general, it attempts to obtain the delivery rate of replication routing and the low resource utilization benefits of forwarding based routing. Spray and Wait achieves resource efficiency by setting a strict upper boundary on the number of copies per message allowed in the network.

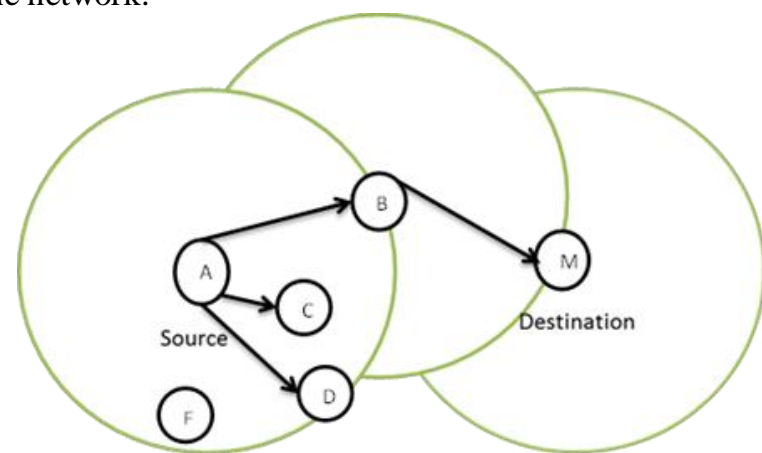

Fig. 2 Sample for Message Path by using Spray and Wait Routing Protocol

Fig. 2 describes the phases of the Spray and Wait routing protocol. Node A wants to send a message to node $\mathrm{M}$ and node $\mathrm{M}$ is not available for node $\mathrm{A}$, so it forwards it to $\mathrm{L}=3$ nodes, which are $\mathrm{B}, \mathrm{C}, \mathrm{D}$ nodes, then these nodes keep the message until they find a direct connection to the destination node. In the above figure, node $\mathrm{B}$ finds a direct connection with the destination node, so it forwards the message to $\mathrm{M}$. 
The Spray and Wait protocol contains two phases. The first is called Spray and the second is called Wait. Spray is where the message is created in the system, a number of L nodes would be attached this message. $\mathrm{L}$ nodes would indicate the maximum number of copies that are allowed to be distributed from the created message in this network. Thus, the source distributes one copy to the L nodes called "relays". The second stage starts when the message is received by the relays, then they start waiting for the particular message until it directly accesses the destination node [11]. In other words, they would hand the message to the destination node only.

Spyropoulos, Psounis and Raghavendra observed that the Spray and Wait routing protocol is very simple but it gave a very beneficial performance and it also gave better performance than all other routing protocols used with DTNs routing network in terms of the number of message transmissions and the delivery delay. Furthermore, it is scalable to the size of the network and it is also scalable to the level of increasing the connectivity.

\section{DESCRIPTION OF THE WORK}

We divide our work in three major parts. First we describe how the virtual map was collected. Second we explain how the meeting points, bus and underground lines were gathered and processed Third we define our experimental scenarios that and explain how our parameters are set.

\section{A. London Olympic area Maps}

We describe how we use novel realistic maps with different connectivity and mobility patterns compared to the ones typically used (Helsinki): We use the London Olympic area because it is significantly more challenging and demanding. We use Open Street Map [6] [Fig 3.] and convert this map to Well-known text format (WKT) [7], which is the only format accepted by the ONE simulator.

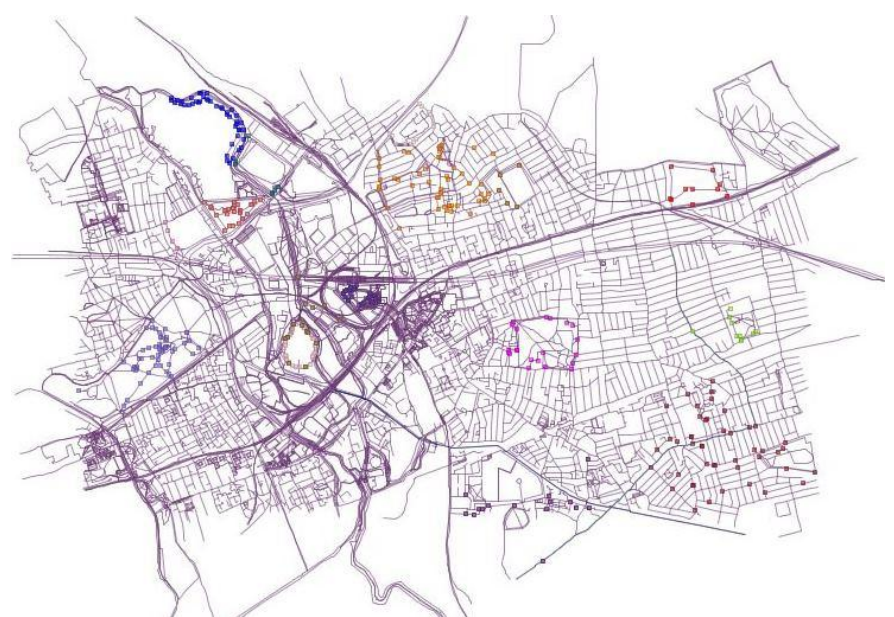

Fig. 3 The London Olympic Area in WKT format

\section{B. Bus and Underground lines}

The gather traces of buses which run through the London Olympic from transport website [8] and from the Google map website [9]. The traces are then transferred to the bus and underground routes one ONE via OpenJUMP software. The following is a list of bus and underground lines that are used on the one simulator for the selected area. Not all the bus lines are used, because some lines do not have a significant effect neither the map nor nodes:

- $\quad$ London Overground Lines (National Rail)

- Docklands Light Railway (DLR)

- Jubilee Line

- Hammersmith and District Line

- Bus 276 /Bus 25 /Bus 158 /Bus 425

- Bus 56 /Bus 69 /Bus 238 /Bus 473

- $\quad$ Bus N73 /Bus 97 /Bus 257 /Bus 488

- Bus $309 \quad$ /Bus N15 /Bus 108

\section{Meeting Points}

We select major meeting points on the map from the London Olympic area. The places are defined by their longitudes and latitude by using the map and converted to WKT by using "OpenJUMP" software [10]. The 
following is a list of places which have been put in separate files from the virtual map and added to the Open JUMP map:

- Place Map.

- East London Cemetery.

- Olympic Stadium.

- Olympic Park.

- City of London Cemetery and Crematorium.

- East Marsh.

- Hackney Marshes.

- Mabley Green.

- Manor Park Cemetery.

- Newham University Hospital.

- St Patrick Catholics Cemetery.

- Victoria Park.

- Wenstead Flats.

- Westfield Stratford City.

- West Ham Cemetery.

- West Ham Park.

- Wick Community Woodland.

- Widnage Park and Plashet Park.

\section{Experiment Scenario}

There are two types of experimental application scenarios which have been used in this paper to obtain more accurate results for evaluating the Prophet, Epidemic and Spray and Wait routing protocols. These application scenarios are explained below:

1) Random Scenario $(\boldsymbol{R S})$ : We assume there are six groups of people and those groups move randomly around the map, but they use only the roads that are defined in the map, We provide bus, tram and underground lines in this area as public transportation. We also support that different groups of people and each person can have different priorities for various interest/meeting points.

2) The Work Day Movement Scenario (WDM): This scenario is more realistic than the random scenario. This kind of scenario can be used with the latest version (ONE1.4.1 []) of the ONE simulator. This kind of implementation requires details such as points on the map for nodes to start their days which are used as houses. Although, it provides a beneficial opportunity to simulate people's daily routine, it still has some limitations. As an example of its limitations, the relationships and behaviours of the users are not able to be simulated with this kind of implementation. Furthermore, the heterogeneous devices between the nodes and the interface are not supported to execute on the ONE simulator.

Working day movement (WDM) attempts to simulate the typical behaviour of people and this could be done by providing some activities that could be followed by the user [5]. As an example of these activities every user would wake up in the morning, go to work every day, go shopping, meet friends and go out. These activities are defined for each group individually. In addition, the nodes in each group might not communicate with all other nodes on the map but every node would encounter the other nodes in the same group. This kind of behaviour, however, would be useful with the Prophet routing protocol because this protocol relies on the previous encounters between the nodes. The following fig shows how the groups of people are distributed on the map:

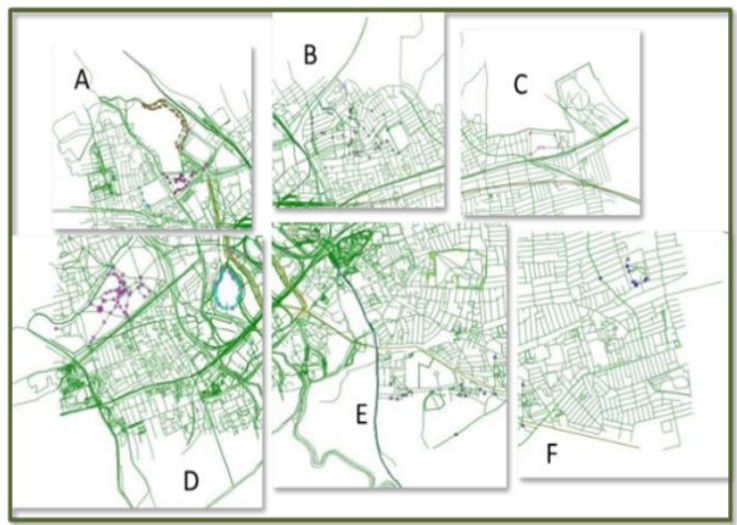

Fig 3 shows how the groups are distributed on the map in WDM 


\section{E. Application}

We provide an application that has the ability to send messages with a random size between 500K$1.5 \mathrm{M}$ and a random short interval between sending messages which is 25 to 35 seconds. Time to live (TTL) defined for any message before it is dropped is twenty four hours. This TTL is selected, because it is assumed that all nodes use Bluetooth and its range, 10 meters. We assume that nodes Bluetooth is ON at all times and reachable by other nodes.

\section{RESULTS AND EVALUATION}

We ran the simulator for both scenarios on our map with various buffer sizes to see the impact of the buffer sizes of DTN routing protocols in the London Olympic map so we select the sizes, 5M, 10M, 15M, 25M, $35 \mathrm{M}, 50 \mathrm{M}$, and $36 \mathrm{M}$.

\section{F. Number of Forwarded}

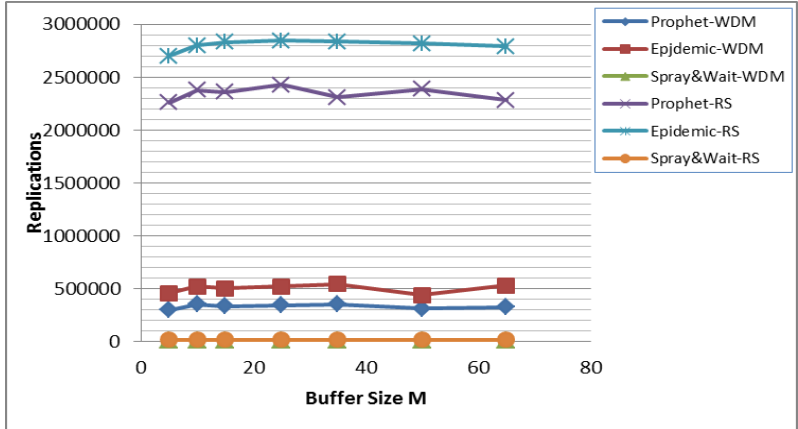

Fig 4 Forwarded for RS \& WDM

Fig 4 illustrates the number of forwarded messages in both RS and WDM scenarios when various buffer sizes are used. It can be clearly seen that the number of forwarded messages in the RS scenario is much higher than the WDM scenario for all buffer size and routing protocols. Furthermore, increasing the buffer size does not have a significant effect on the number of forwarded messages for all protocols used. The first protocol which will be discussed is the Epidemic routing protocol. It has the highest number of forwarded messages in both scenarios although its number of forwarded messages in WDM decreases rapidly in the RS scenario. On the other hand, the Spray and Wait routing protocol has a very low number of forwarded messages for both scenarios and various buffer sizes. Finally, the number of forwarded messages for the Prophet routing protocol is much higher in the RS scenario than in the WDM scenario. Using different buffer sizes does not impact on the number of forwarded messages for both scenarios.

\section{G. Delivered}

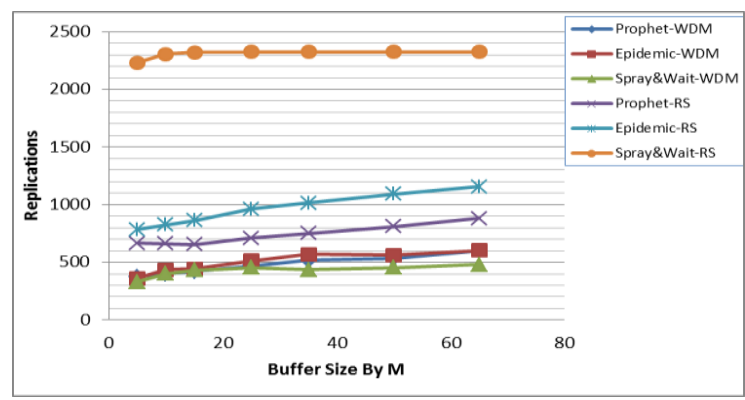

Fig 5 Delivered for RS \& WDM

Fig 5 shows that the Spray and Wait routing protocol delivered a very high number of messages in the RS more than 2,000 from over 2,913 created messages. However, this number of delivered messages is significantly reduced in the WDM scenario with less than 500 messages and the probability to deliver a message for this protocol in WDM is 10 percent. Furthermore, the number of delivered messages by using the Prophet routing protocol increases with both scenarios when the buffer size is increased, but the number of delivered messages with RS is better in the WDM scenario. Additionally, the delivered messages in the Epidemic routing protocol are affected by increasing the buffer size, so it increases rapidly. However its performance with RS is better than with WDM. 


\section{H. Dropped}

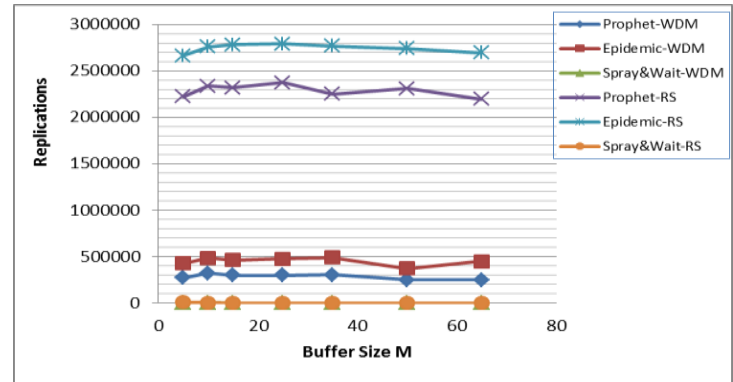

Fig 6 Dropped for RS \& WDM

Figure 6 provides information about running three routing protocols with RS and WDM scenarios and how they are affected by changing the size of the buffer. The first protocol, the Spray and Wait routing protocol drops a small number of messages with all buffer sizes in both scenarios. The second protocol is the Prophet routing protocol. It does not show a significant effect in RS when using various buffer sizes. However, the number of dropped messages has rapidly reduced in the WDM scenario and the number of dropped messages has also decreased when the buffer size increased with the WDM scenario. The third protocol, an Epidemic routing protocol is not affected by changing the buffer size, but it is obviously high with WDM. Furthermore the number of dropped messages is very high with RS at more than 2,500,000 messages whereas the number of dropped messages in the WDM scenario, unlike the RS scenario, is less than 500,000 messages with all buffer sizes.

\section{Overhead Ratio}

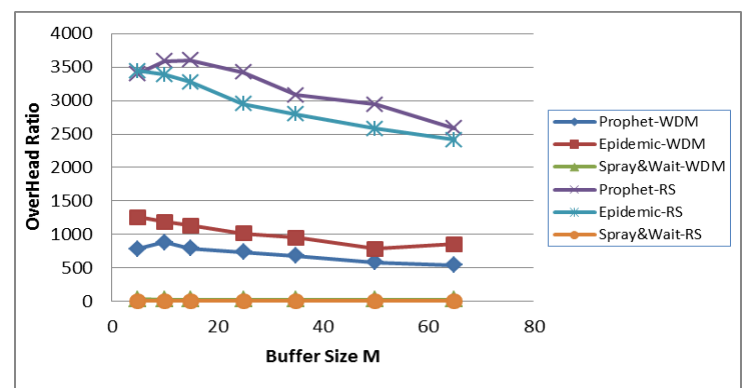

Fig 7 Overhead Ratio for RS \& WDM

Figure 7 shows the overhead ratio for both WDM and RS scenarios. This is used to measure how much the routing protocol cost the resource. So, all routing protocol algorithms attempt to minimize the overhead ratio. The Spray and Wait routing protocol algorithm costs the resource very little in both scenarios. The second routing algorithm is the Prophet routing protocol algorithm which costs the resource almost 500 for all used buffer sizes in WDM, but these changes with an RS scenario is above 2,500 for all used buffer sizes. Furthermore, the changing buffer size has a significant effect on the overhead ratio with RS, and it reduces rapidly when the buffer size is increased. For example, the overhead ratio for the Prophet routing protocol with the buffer size $5 \mathrm{M}$ is 3,400 whereas this value becomes 2,400 with the buffer size $65 \mathrm{M}$. In addition, the overhead ratio for the Epidemic routing protocol is very high with RS scenario and this value is decreased when the buffer size is increased from 3,450 with a buffer size of $5 \mathrm{M}$ to 2,350 with a buffer size of $65 \mathrm{M}$. The overhead ratio for Epidemic routing protocol in the WDM scenario decreases when the buffer size is increased.

\section{J. Latency Average}

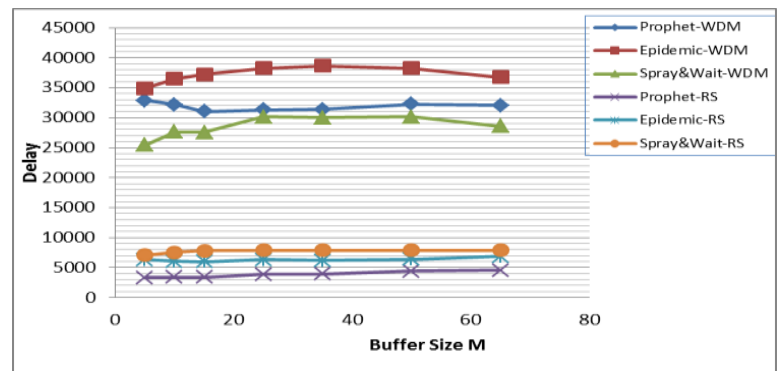

Fig 8 Delay for RS \& WDM 
Fig 8 demonstrates the delayed message time, which is the time taken from the messages being generated by the sender to being received by the destination node, for both RS and WDM scenarios. It shows that the WDM scenario has the higher delay time than the delay in RS scenario for all routing protocols. It can be seen that the Prophet routing protocol in the RS has the lowest delay time, but the second highest routing protocol delay is in the WDM scenario. It also does not show much effect by changing the buffer sizes in both scenarios. The second protocol is the Spray and Wait routing protocol. The delay is very high in WDM at more than 25,000s with all buffer sizes but this value rapidly decreased in the RS scenario, but it is still higher than the other in RS. Finally, the delay in the Epidemic routing protocol is the highest in WDM, but it is the second highest in the RS. It also does not show much effect by increasing the buffer size.

\section{CONCLUSION}

This paper has investigated the performance of a probabilistic routing protocol by using the virtual real map for the London Olympic area. We proposed two mobility scenarios which are Work Day Movement scenario (WDM) and Random scenario (RS) that allow us to better understanding the impact of the mobility models in highly congested urban areas on a range of DTN routing protocols. We simulate people's daily lives by providing for any node a house location, an office location and meeting locations on the map to allow users to meet their friends and have different preferences, whereas the second scenario allow random sending and receiving policy for messages among the nodes that move via shortest paths between any two locations. We used probabilistic resource aware protocol, epidemic and spray and wait protocols where each one was run seven times, each time with different buffer sizes in an attempt to observe the impact of changing the buffer size on the performance of each protocol.

After gathering the results from the simulator, we compared the performance of resource aware probabilistic protocol with the others in terms of number of forwarded messages, delivered messages, dropped messages, overhead ratio and latency average. We observed that the performance of the resource aware probabilistic protocol in the WDM scenario was better than its performance with the random scenario, because the WDM scenario is much closer to users' lives, so the nodes meet each other much more than in the RS scenario. However, the overhead ratio, and dropped messages with the probabilistic resource aware protocol are still much higher than Spray and Wait in the WDM scenario.

We used a realistic map and real bus and underground traces while people still moved randomly. Also, sender and destination are selected by the simulator randomly. We aim to conduct further research by providing a real movement people movement and communication patterns ( e.g. by utilising San Francisco map [11] and analysis of users; tweets). We aim to do a real life deployment of our protocol over mobile phones in the city centre of a populated city to evaluate the success of this alternative method of communication.

\section{REFERENCES}

[1] J. Votano, M. Parham and L. Hall, "A review of current routing protocols for ad hoc mobile wireless networks," IEEE Personal Communications Magazine, vol. 6, no. 2, p. 46-55, Apr. 1999.

[2] T. Spyropoulos, K. Psounis and C. S. Raghavendra, "Spray and wait: an efficient routing scheme for intermittently connected mobile networks," New York, 2005.

[3] A. Vahdat and D. Becker, "Epidemic Routing for Partially-Connected Ad Hoc Networks," Duke University, 2000.

[4] A. Lindgren, A. Doria and O. Schelen, "Probabilistic Routing in Intermittently Connected Networks," ACM SIGMOBILE Mobile Computing and Communications Review, vol. 7, no. 3, pp. 19 - 20, 2003.

[5] Barun, "The One," 2011. [Online]. Available: http://delay-tolerant-networks.blogspot.co.uk/p/one-tutorial.html. [Accessed 03 June 2012].

[6] "The One," 2007. [Online]. Available: http://www.netlab.tkk.fi/tutkimus/dtn/theone. [Accessed 05 June 2012].

[7] CCBY-SA, "Open Street Map," 2012. [Online]. Available: http://www.openstreetmap.org/. [Accessed 01 June 2012].

[8] T. f. L. Team, "London Transport," 2012. [Online]. Available: http://www.tfl.gov.uk/tfl/gettingaround/maps/buses/. [Accessed 2007 2012].

[9] Google, "Google Maps," 2012. [Online]. Available: https://maps.google.com. [Accessed 1507 2012].

[10] V. Solutions, "OPEN JUMP," 2011. [Online]. Available: http://www.openjump.org/. [Accessed 01 June 2012].

[11] C. team, "epfl/mobility(v. 2009-02-24)," CRAWDAD , $24 \quad 2 \quad 2009 . \quad$ [Online]. Available: http://crawdad.cs.dartmouth.edu/meta.php?name=epfl/mobility\#N100F7. [Accessed 015 2012].

[12] M. Grossglauser and D. Tse, "Mobility increases the capacity of ad-hoc wireless networks," IEEE/ACM Transactions on Networking, vol. 10, no. 4, pp. 477- 486, 2002.

[13] C.-C. Shen, G. Borkar, S. Rajagopalan and C. Jaikaeo, "Interrogation-based relay routing for ad hoc satellite networks," Global Telecommunications Conference, 2002. GLOBECOM '02. IEEE, vol. 3, pp. 2920- 2924, 2002.

[14] C. P. Mayer, "osm2wkt - OpenStreetMap to WKT Conversion," 2010. [Online]. Available: http://www.tm.kit.edu/ mayer/osm2wkt. [Accessed 01 June 2012].

[15] A. Keränen, J. Ott and T. Kärkkäinen, "The ONE Simulator for DTN Protocol Evaluation," Brussels, 2009. 